Research Paper

\title{
High pretreatment serum CA19-9 level predicts a poor prognosis for patients with stage III colon cancer after curative resection and adjuvant chemotherapy
}

\author{
Wenhao Zhou ${ }^{1 *}$, Fan Yang ${ }^{2}$, Jianhong Peng1 ${ }^{*}$, Fulong Wang1, Yuzhu Lin ${ }^{1}$, Wu Jiang1, Xia Yang1, Liren Li1, \\ Zhenhai Lu' ${ }^{1}$, Desen Wan ${ }^{1}$, Zhizhong Pan ${ }^{\bowtie}$, Wenhua Fan ${ }^{1 凶}$ \\ 1. Department of Colorectal Surgery, Sun Yat-sen University Cancer Center; State Key Laboratory of Oncology in South China; Collaborative Innovation \\ Center for Cancer Medicine, Guangzhou, 510060, P.R. China \\ 2. Department of Gynecologic Oncology, Sun Yat-sen University Cancer Center; State Key Laboratory of Oncology in South China; Collaborative Innovation \\ Center for Cancer Medicine Guangzhou, 510060, P.R. China \\ *Wenhao Zhou, Fan Yang and Jianhong Peng contributed equally to this work.
}

$\triangle$ Corresponding authors: Wenhua Fan and Zhizhong Pan, Department of Colorectal Surgery, Cancer Center, Sun Yat-sen University, 651 Dongfeng Road East, Guangzhou, P. R. China, 510060. Tel.: +86-20-87343609; Fax: +86-20-87343609; E-mail: fanwh@sysucc.org.cn

(c) Ivyspring International Publisher. This is an open access article distributed under the terms of the Creative Commons Attribution (CC BY-NC) license (https://creativecommons.org/licenses/by-nc/4.0/). See http://ivyspring.com/terms for full terms and conditions.

Received: 2018.11.09; Accepted: 2019.05.10; Published: 2019.06.09

\begin{abstract}
Carbohydrate antigen 19-9 (CA19-9) is one of the most widely used tumor markers in gastrointestinal cancer. However, serum CA19-9 is not a recommended routine measurement in colon cancer. In this study, we evaluated the value of the preoperative serum CA19-9 level for the prediction of postoperative prognosis in stage III colon cancer. The medical records of 367 consecutive patients with stage III colon cancer who underwent curative resection followed by adjuvant chemotherapy with oxaliplatin and capecitabine between December 2007 and April 2015 were retrospectively reviewed. We determined the optimal cutoff value of CA19-9 for 3-year recurrence using the receiver operating characteristic (ROC) method. Differences in disease-free survival (DFS) and overall survival (OS) rates stratified by CA19-9 level were compared by using Kaplan-Meier and log-rank tests. A Cox proportional hazards model was used to identify prognostic variables for DFS and OS. The statistically determined best cutoff value for CA19-9 was $24 \mathrm{U} / \mathrm{ml}$. High CA19-9 levels (> $24 \mathrm{U} / \mathrm{ml}$ ) were significantly associated with poorly differentiated tumors, abnormal carcinoembryonic antigen (CEA) levels, and a high cumulative incidence of lung metastasis. Additionally, compared with low CA19-9 levels, high preoperative CA19-9 levels were associated with inferior 3-year DFS and OS rates, especially for high-risk patients (T4Nany or TanyN2). Multivariate analyses revealed that CA19-9 was an independent factor associated with both DFS (hazard ratio [HR], 2.248; 95\% confidence interval [Cl], 1.393-3.628; $\mathrm{P}=0.001$ ) and OS (HR: 2.081; $95 \% \mathrm{Cl}: 1.137-3.808 ; \mathrm{P}=0.017)$. The results of this study showed that high levels of preoperative serum CA19-9 indicated a worse prognostic outcome for stage III colon cancer patients. An early follow-up protocol to assess lung metastasis and a full course of adjuvant chemotherapy should be used for these patients.
\end{abstract}

Key words: CA19-9, colon cancer, stage III, prognosis

\section{Introduction}

Colorectal cancer (CRC) has been one of the leading malignant tumors that threaten human health in both the United States and China [1, 2]. At diagnosis, approximately one-third of patients exhibit regional lymph node metastasis and stage III colon cancer $[3,4]$. As a standard of care, curative tumor resection combined with adjuvant chemotherapy is well established and widely accepted for the 
treatment of patients with stage III colon cancer $[5,6]$. Due to improvements in adjuvant chemotherapy, the 5 -year relative survival rate for patients with stage III colon cancer increased from 55\% during the years $1975-1977$ to $74 \%$ during the years 2006-2012 [7]. However, not all patients derived benefit from curative-intent adjuvant chemotherapy; $20 \%$ to $30 \%$ of patients ultimately developed distant metastases [8, 9]. Herein, an abbreviated shortened duration of adjuvant therapy might be a feasible way to avoid or reduce toxicities in some patients without impairing oncologic outcomes. As a result, the adjuvant chemotherapy needs an optimization of the duration according to the post-operative recurrence risk of the patients. It was necessary to explore effective prognostic markers for risk stratification and the further individualization of postoperative follow-up and postoperative therapy regimens.

Peripheral blood can be obtained noninvasively through a simple procedure that is easily accepted by patients. Therefore, the detection of serum tumor biomarkers was in line with the requirements of tumor specificity and the principle of noninvasive examination. Serum carbohydrate antigen 19-9 (CA19-9) has been known as a tumor biomarker in colon carcinoma for almost 40 years [10]. In addition, CA 19-9 is secreted by normal biliary epithelium, and its level can be markedly elevated because of benign biliary disease and inflammatory processes [11, 12]. To date, serum CA19-9 is one of the most frequently applied tumor biomarkers in gastrointestinal cancers and is used particularly for detecting and monitoring pancreatic cancer [13, 14] and bile duct cancer [15]. However, the current guidelines of the European Society of Medical Oncology (ESMO) and the National Comprehensive Cancer Network (NCCN) recommended the routine measurement of preoperative CEA levels but not of CA19-9 levels for postoperative surveillance [5, 16]. In fact, previous studies have demonstrated that preoperative CA19-9 levels can be used as an additional marker to monitor the disease process after CRC resection in patients who do not exhibit an increased CEA level [17, 18]. Nevertheless, evidence for the prognostic or predictive values of the preoperative CA19-9 level in patients with stage III colon cancer receiving curative treatment remains unavailable. Accordingly, we hypothesized that the preoperative serum CA19-9 level was able to divide patients with stage III colon cancer into subgroups with different prognoses.

Therefore, the current study aimed to determine whether preoperative serum CA19-9 levels presented prognostic value for stage III colon cancer patients undergoing curative resection followed by oxaliplatin-containing adjuvant chemotherapy.
Accordingly, we determined the optimal CA19-9 cutoff value and subsequently evaluated the association of CA19-9 level with postoperative metastatic patterns and long-term survival rates in order to stratify risk and optimize postoperative therapy.

\section{Patients and Methods}

\section{Patient selection}

In this study, the medical records of 450 consecutive patients were reviewed. All patients were diagnosed with stage III colon cancer and underwent tumor resection and followed by adjuvant chemotherapy between December 2007 and April 2015 at Sun Yat-sen University Cancer Center. Patients who met the following inclusion criteria were enrolled: (1) histologically confirmed adenocarcinoma; (2) American Society of Anesthesiologists (ASA) class I-II status; (3) performance of a complete curative resection for colon tumor; (4) treatment with XELOX adjuvant chemotherapy (oxaliplatin and capecitabine); (5) absence of preoperative anticancer treatment; and (6) a postoperative follow-up period of at least 3 months. The patient demographics, tumor characteristics, and adjuvant chemotherapy cycles were carefully recorded. The tumor stage was classified according to the 2010 American Joint Committee on Cancer staging system. All procedures were performed according to the ethical standards of the 2013 World Medical Association Declaration of Helsinki. The informed consent requirement was waived based on the nature of this retrospective study, in which patient data was kept confidential. This study was approved by the Institutional Review Board and Independent Ethics Committee of Sun Yat-sen University Cancer Center.

\section{Perioperative evaluation and treatment}

Preoperative blood tests, including measurement of CA19-9 and CEA levels, at the time of diagnosis were carried out within 2 weeks of tumor resection. Preoperative imaging by contrast-enhanced computed tomography (CT) of the chest, abdomen, and pelvis was performed to confirm the preoperative stage of the patients. All patients underwent standard complete mesocolic excision (CME) with regional lymphadenectomy for colon tumor. XELOX adjuvant chemotherapy with $130 \mathrm{mg} / \mathrm{m}^{2}$ oxaliplatin administered intravenously on Day 1 and 1000 $\mathrm{mg} / \mathrm{m}^{2}$ capecitabine administered orally twice daily on Days 1-14 for a 3-week cycle was recommended to begin 3-6 weeks after surgery. The postoperative tumor pathological staging was confirmed by two independent pathologists. Patients with tumors staged as T4Nany or TanyN2 were classified as the 
high-risk group, while patients with tumors staged as T1-3 and N1 were classified as the low-risk group.

\section{Follow-up}

Follow-up data were recorded from the tracking system of our center. Patients were monitored through subsequent visits every 3 months for the first 2 years and then semiannually for 5 years after tumor resection. Clinical examination, assessment of CEA and CA19-9 levels, and chest radiography were conducted every 3 months. Chest/abdominal/pelvic $\mathrm{CT}$ and colonoscopy were performed annually. Overall survival (OS) was defined as the interval from the date of tumor resection to the date of death from any cause or to the last follow-up. Disease-free survival (DFS) was defined as the interval from tumor resection to the date of disease recurrence, death or the last follow-up. The final follow-up visit occurred in April 2018.

\section{Statistical analysis}

The optimal CA19-9 cutoff values for the prediction of 3-year DFS and OS were identified by receiver operating characteristic (ROC) curve analysis according to the highest Youden index by using the Cutoff Finder software program (http://molpath.charite.de/cutoff/). The rest of the data were analyzed using IBM SPSS statistics software, version 21.0 (IBM Corp., Armonk, NY, USA) and GraphPad Prism version 6.01 (GraphPad, Inc., La Jolla, CA, USA). Comparisons between the groups of patients with high and low levels of CA19-9 according to the clinicopathological variables were performed with chi-squared or Fisher's exact tests. The OS and RFS rates were estimated with the Kaplan-Meier method, and the differences between groups were then assessed with the log-rank test. Parameters for which $\mathrm{P}<0.05$ for OS and DFS in the univariate Cox models were further assessed in multivariate Cox models. Hazard ratios (HRs) and 95\% confidence intervals (CIs) were subsequently calculated. All of the statistical tests were two-sided; $\mathrm{P}<0.05$ was considered significant.

\section{Results}

\section{Patient selection and characteristics}

Of the 450 patients, 83 patients were excluded for the following reasons: treatment with other regimens of adjuvant chemotherapy ( $n=54)$; follow-up of less than 3 months $(\mathrm{n}=11)$ and incomplete tumor resection $(n=18)$. Overall, 367 eligible patients were identified for analysis in the current study. Of these patients, 211 (57.5\%) were male, and $156(42.5 \%)$ were female, with a median age of 56 years (range, 19-85 years). The clinicopathological and treatment data regarding all eligible patients are summarized in Table 1. The median numbers of resected and metastatic lymph nodes were 15 (range, 2-63) and 2 (range, 1-23), respectively. Regarding risk classification, 218 (59.4\%) patients were included in the high-risk group, while 149 patients were included in the low-risk group. In total, 9 (2.4\%) patients experienced postoperative complications, including 7 $(1.9 \%)$ patients with intestinal obstruction, $1(0.3 \%)$ with anastomotic leakage, and $1(0.3 \%)$ with infection at the incision site. The median number of cycles of XELOX adjuvant chemotherapy for all of the patients was 6 (range, 1-8).

Table 1. Clinicopathological characteristics of the patients

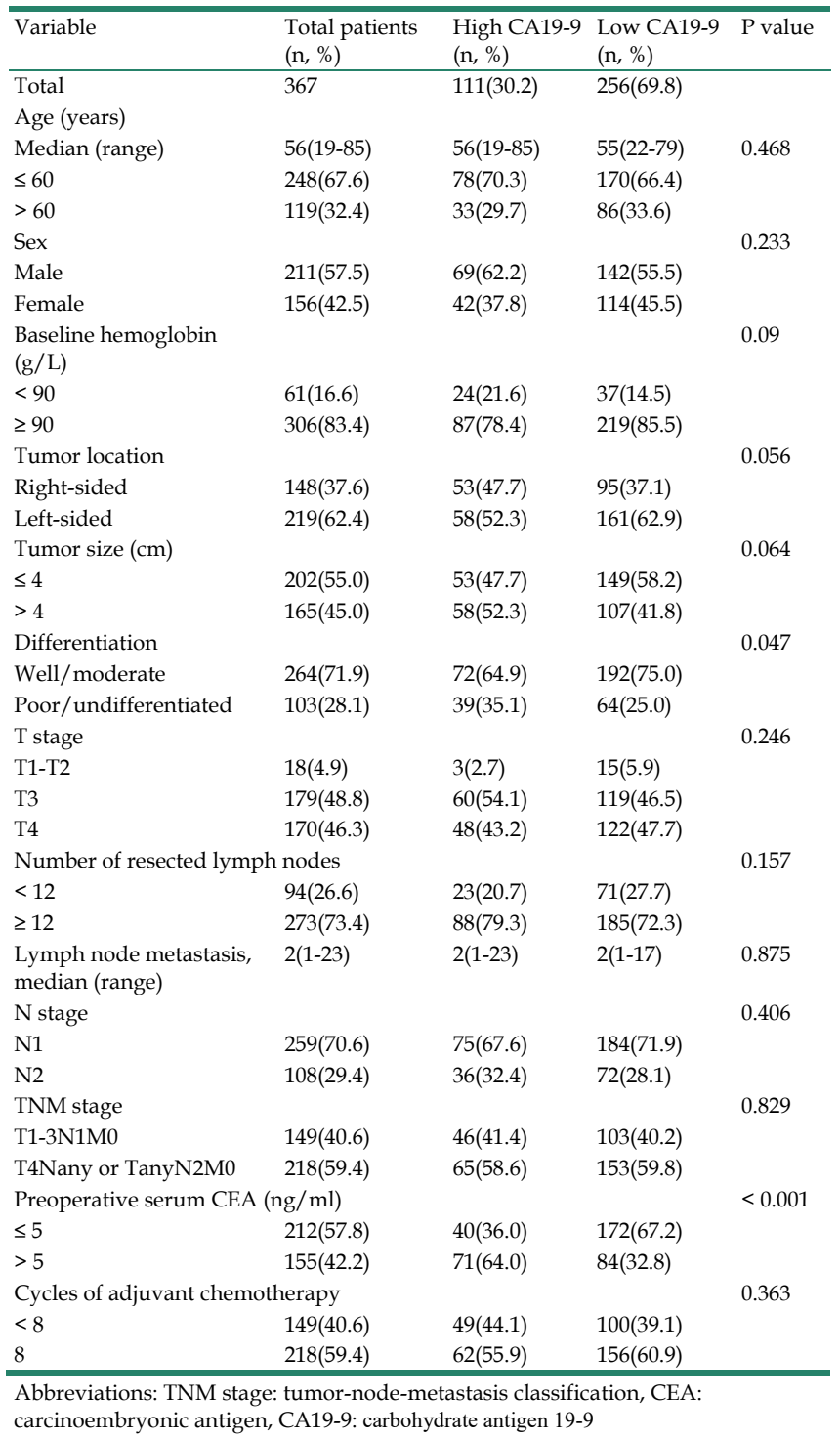

\section{Cutoff points for CA19-9}

According to ROC analysis, the optimal CA19-9 cutoff value for 3-year DFS was 23.64, with the highest Youden index (0.253), a sensitivity of $51.5 \%$ and a 
specificity of $73.8 \%$ (area under the ROC curve [AUC], 0.600; 95\% CI, 0.521-0.677; $\mathrm{P}=0.012$; Figure 1A). The optimal CA19-9 cutoff value for 3-year OS was 26.98, with the highest Youden index (0.278), a sensitivity of $53.3 \%$ and a specificity of $74.5 \%$ (AUC, $0.630 ; 95 \%$ CI, $0.519-0.639$; $\mathrm{P}=0.019$; Figure $1 \mathrm{~B})$. Thus, patients were divided into the high-CA19-9 group ( $\mathrm{n}=111,30.2 \%)$ and the low-CA19-9 group ( $\mathrm{n}=256,69.8 \%$ ) based on the CA19-9 cutoff value of $24 \mathrm{U} / \mathrm{ml}$.

\section{Relationships between CA19-9 level and clinicopathological characteristics}

Relationships between CA19-9 level and different clinicopathological characteristics are presented in Table 1. High CA19-9 levels had a significantly greater association with poorly differentiated and undifferentiated tumors than did low CA19-9 levels (35.1\% vs. $25.0 \%, \mathrm{P}=0.047)$. In addition, patients with a high CA19-9 level were more likely than patients with a low CA19-9 level to exhibit an abnormal CEA level (64.0\% vs. $32.8 \%$; P < 0.001). No significant differences were observed between patients with low and high CA19-9 levels in terms of age, sex, baseline hemoglobin, tumor location, TNM stage, or number of cycles of adjuvant chemotherapy.

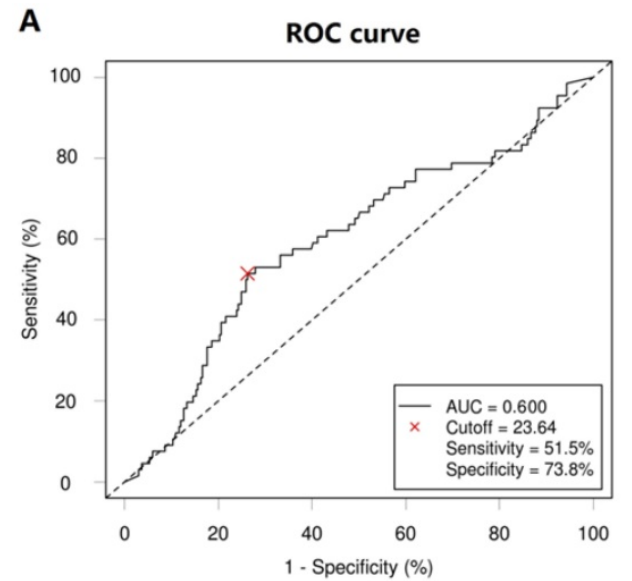

\section{Analysis of CA19-9 level and postoperative metastasis}

The median follow-up period for all patients was 58.7 months (range, 7.5-115.0 months). During this period, $75(20.4 \%)$ patients experienced tumor metastasis; in total, $43(11.7 \%)$ patients died of tumor progression. The 3-year DFS and OS rates were $84.3 \%$ and $92.5 \%$, respectively, in the entire study population. The relationships between CA19-9 level and postoperative metastasis are shown in Table 2. Patients with a high CA19-9 level presented a significantly higher risk of total postoperative metastasis $(32.4 \%$ vs. $15.2 \%, \mathrm{P}<0.001)$, lung metastasis $(12.6 \%$ vs. $3.9 \%, P=0.002)$ and abdominopelvic metastasis $(10.8 \%$ vs. $3.5 \%, \mathrm{P}=0.006)$ than those in the low CA19-9 group. The cumulative incidences of liver $(8.7 \%$ vs. $4.5 \%, \mathrm{P}=0.212)$ and abdominopelvic metastasis $(7.4 \%$ vs. $3.3 \%, \mathrm{P}=0.094)$ within 3 years were not significantly different between the high CA19-9 and low CA19-9 groups (Figure 2A and 2C). However, the cumulative incidence of lung metastasis within 3 years was threefold higher in the high-CA19-9 group than in the low-CA19-9 group $(12.0 \%$ vs. $3.9 \%, \mathrm{P}<0.001$, Figure $2 \mathrm{~B})$; this difference was statistically significant.

B

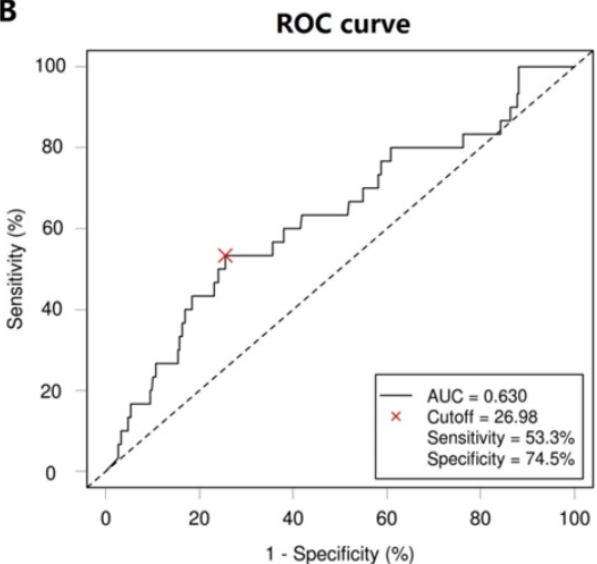

Figure 1. Receiver operating characteristic (ROC) curve for carbohydrate antigen 19-9 (CA19-9) level according to 3-year (A) disease-free survival (DFS) and (B) overall survival (OS). The dashed line from the left bottom to the top right corners represents a random rate regardless of the positive and negative base rates.

A

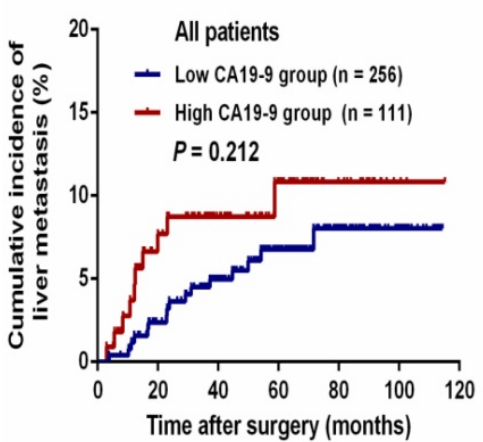

B

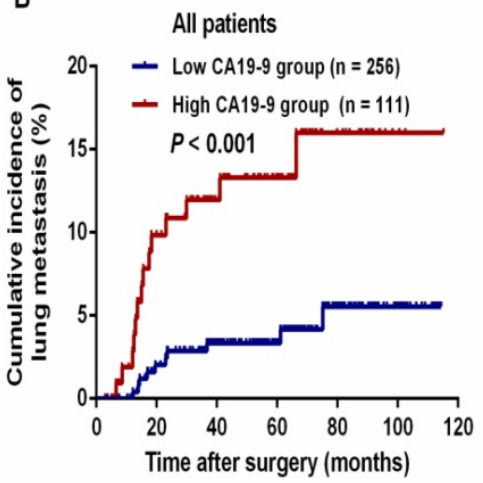

C

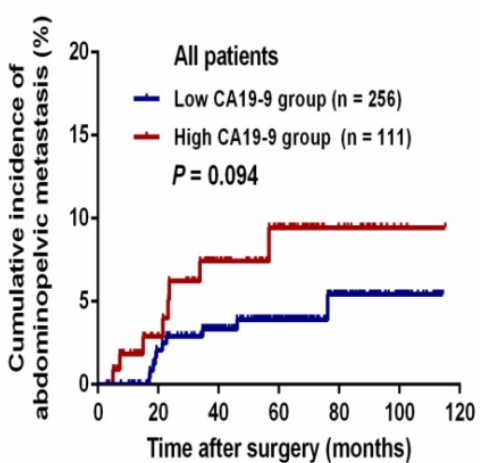

Figure 2. The cumulative incidence of postoperative metastasis in patients with stage III cancer who underwent curative treatment: (A) cumulative incidence of liver metastasis, (B) cumulative incidence of lung metastasis, and (C) cumulative incidence of abdominopelvic metastasis. 
A

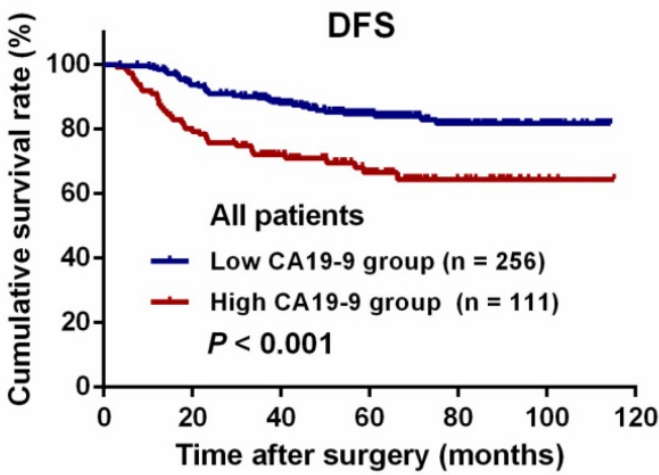

B

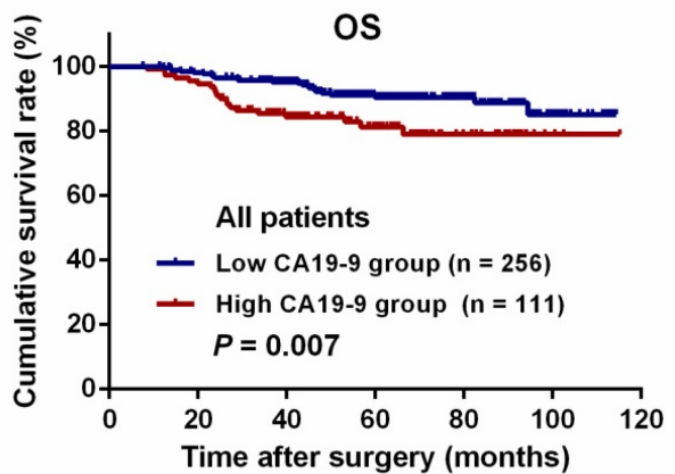

Figure 3. Kaplan-Meier long-term survival curves grouped by high and low preoperative serum carbohydrate antigen 19-9 (CA19-9) levels. (A) Disease-free survival (DFS) of all patients. (B) Overall survival (OS) of all patients.

Table 2. Postoperative metastatic patterns of patients after curative resection

\begin{tabular}{lllll}
\hline Variable & $\begin{array}{l}\text { Total patients } \\
(\mathrm{n}, \%)\end{array}$ & $\begin{array}{l}\text { High CA19-9 }\left(\mathrm{n}=\begin{array}{l}\text { Low CA19-9 }(\mathrm{n}= \\
111, \%)\end{array}\right. \\
256, \%)\end{array}$ & $\begin{array}{l}\mathrm{P} \\
\text { value }\end{array}$ \\
\hline $\begin{array}{l}\text { Postoperative } \\
\text { metastasis }\end{array}$ & & & & $<$ \\
Yes & $75(20.4)$ & $36(32.4)$ & $39(15.2)$ & 0.001 \\
No & $292(80.7)$ & $75(67.6)$ & $217(84.8)$ & \\
Liver metastasis & & & & 0.344 \\
Yes & $26(7.7)$ & $10(5.5)$ & $16(6.3)$ & \\
No & $341(92.3)$ & $101(94.5)$ & $240(93.8)$ & \\
Lung metastasis & & & & 0.002 \\
Yes & $24(6.5)$ & $14(12.6)$ & $10(3.9)$ & \\
$\begin{array}{l}\text { No } \\
\text { Abdominopelvic } \\
\text { metastasis }\end{array}$ & $343(93.5)$ & $97(87.4)$ & $246(96.1)$ & \\
Yes & & & & \\
No & $21(5.7)$ & $12(10.8)$ & $9(3.5)$ & \\
\hline
\end{tabular}

Abbreviations: CA19-9: carbohydrate antigen 19-9

\section{Association between CA19-9 level and survival outcome}

For all patients, the 3-year DFS and OS rates were significantly lower in the high-CA19-9 group than in the low-CA19-9 group (DFS: $72.0 \%$ vs. $89.7 \%$, $\mathrm{P}<0.001$, Figure $3 \mathrm{~A}$; OS: $85.5 \%$ vs. $95.6 \%, \mathrm{P}=0.007$, Figure 3B). There were no differences in the DFS and OS rates among the patients in the low-risk group with high and low CA19-9 levels (DFS: $75.8 \%$ vs. $90.1 \%, \mathrm{P}=0.065$, Figure $4 \mathrm{~A}$; OS: $93.3 \%$ vs. $96.0 \%, \mathrm{P}=$ 0.855 , Figure $4 \mathrm{~B})$. However, among the patients in the high-risk group, the 3-year DFS and OS rates were significantly lower in the high-CA19-9 group than in the low-CA19-9 group (DFS: $69.2 \%$ vs. $89.4 \%, \mathrm{P}<$ 0.001, Figure 4C; OS: $79.9 \%$ vs. $95.4 \%, \mathrm{P}=0.002$, Figure 4D).

As shown in Table 3, the univariate analysis revealed that high CA19-9 level (HR: 2.468; 95\% CI: 1.569-3.884; $\mathrm{P}<0.001$ ), high CEA level (HR: 1.629; 95\% CI: 1.035-2.565; $\mathrm{P}=0.035$ ), and male sex (HR: 1.745; 95\% CI: 1.074-2.835; $\mathrm{P}=0.025)$ were the significant negative predictors of 3-year DFS. In the multivariate analysis, CA19-9 level (HR: 2.248; 95\% CI: 1.393-3.628;
$\mathrm{P}=0.001$ ) and sex (HR: 1.752; 95\% CI: 1.078-2.849; $\mathrm{P}=$ $0.024)$ were identified as independent predictors of 3-year DFS.

Table 3. Univariate and multivariate analyses of prognostic factors for disease-free survival for all patients

\begin{tabular}{|c|c|c|c|c|}
\hline & Univariate analysis & & Multivariate analy & \\
\hline Variable & $\mathrm{HR}(95 \% \mathrm{CI})$ & P value & HR (95\% CI) & $\begin{array}{l}\mathrm{P} \\
\text { value }\end{array}$ \\
\hline Age, years (> 60 vs. $\leq 60$ ) & $1.552(0.980-2.457)$ & 0.061 & & \\
\hline Sex (Male vs. Female) & $1.745(1.074-2.835)$ & 0.025 & $1.752(1.078-2.849)$ & 0.024 \\
\hline $\begin{array}{l}\text { Baseline hemoglobin, } \mathrm{g} / \mathrm{l} \\
(<90 \text { vs. } \geq 90)\end{array}$ & $0.854(0.450-1.619)$ & 0.628 & & \\
\hline $\begin{array}{l}\text { Tumor location } \\
\text { (Right-sided vs. Left-sided }\end{array}$ & $1.102(0.696-1.746)$ & 0.678 & & \\
\hline $\begin{array}{l}\text { Tumor size, cm (> } 4 \text { vs. } \leq \\
\text { 4) }\end{array}$ & $0.729(0.458-1.161)$ & 0.184 & & \\
\hline $\begin{array}{l}\text { Differentiation (Poor vs. } \\
\text { Well/ moderate) }\end{array}$ & $1.255(0.768-2.052)$ & 0.365 & & \\
\hline T stage (T4 vs. T1-3) & $1.243(0.788-1.963)$ & 0.350 & & \\
\hline $\begin{array}{l}\text { Number of resected } \\
\text { lymph nodes (<12 vs. } \geq 12 \\
\text { ) }\end{array}$ & $1.364(0.840-2.216)$ & 0.210 & & \\
\hline N stage (N2 vs. N1) & $1.388(0.866-2.225)$ & 0.174 & & \\
\hline $\begin{array}{l}\text { Preoperative CEA, ng/ml } \\
(>5 \text { vs. } \leq 5)\end{array}$ & $1.629(1.035-2.565)$ & 0.035 & $1.317(0.816-2.127)$ & 0.260 \\
\hline $\begin{array}{l}\text { Preoperative CA19-9, } \\
\mathrm{U} / \mathrm{ml}(>24 \text { vs. } \leq 24 \text { ) }\end{array}$ & $2.468(1.569-3.884)$ & $<0.001$ & $2.248(1.393-3.628)$ & 0.001 \\
\hline $\begin{array}{l}\text { Adjuvant chemotherapy, } \\
\text { number of cycles (8 vs. < } \\
\text { 8) }\end{array}$ & $0.748(0.474-1.180)$ & 0.212 & & \\
\hline
\end{tabular}

As shown in Table 4, the univariate analysis revealed that high CA19-9 level (HR: 2.231; 95\% CI: 1.224-4.067; $\mathrm{P}<0.001$ ), right-sided colon cancer (HR: 2.034; 95\% CI: 1.114-3.716; $\mathrm{P}=0.021)$, T4 stage (HR: 1.926; 95\% CI: $1.022-3.628 ; \mathrm{P}=0.043)$, and N2 stage (HR: 1.885 ; 95\% CI: 1.031-3.445; P $=0.039$ ) were the significant negative predictors of 3-year OS, while 8 cycles of adjuvant chemotherapy (HR, $0.510 ; 95 \% \mathrm{CI}$, $0.279-0.930 ; \mathrm{P}=0.028$ ) was the significant protective factor for 3-year OS. In the multivariate analysis, CA19-9 level (HR: 2.081; 95\% CI: 1.137-3.808; P = 0.017), tumor location (HR: 1.902; 95\% CI: 1.038-3.485; 
$\mathrm{P}=0.038)$, T stage (HR: 1.949; 95\% CI: 1.028-3.697; $\mathrm{P}=$ $0.041)$, and number of cycles of adjuvant chemotherapy (HR: 0.510; 95\% CI: 0.297-0.934; $\mathrm{P}=$ $0.029)$ were identified as independent predictors of 3-year OS.

\section{Discussion}

Our study evaluated the prognostic value of the preoperative serum CA19-9 level in stage III colon cancer patients receiving curative treatment. In total, we presented three major findings. First, we found that a high preoperative CA19-9 level indicated a higher risk of postoperative lung metastasis. Furthermore, our final results also revealed a significant correlation between elevated pretreatment serum CA19-9 levels and poor prognosis in stage III colon cancer patients, especially high-risk patients. Finally, our multivariate analysis identified a high preoperative CA19-9 level as an independent prognostic factor for 3-year OS and DFS. To the best of our knowledge, the current study is the first to demonstrate the prognostic value of the preoperative CA19-9 level in patients with curatively resected stage III colon cancer treated with the XELOX regimen.
Table 4. Univariate and multivariate analyses of prognostic factors for overall survival for all patients

\begin{tabular}{|c|c|c|c|c|}
\hline & Univariate analysis & & Multivariate a & analysis \\
\hline Variable & HR $(95 \%$ CI) & $P$ value & HR $(95 \%$ CI) & P value \\
\hline $\begin{array}{l}\text { Age, years (> 60 vs. } \leq 60 \\
\text { ) }\end{array}$ & $1.425(0.773-2.628)$ & 0.256 & & \\
\hline Sex (Male vs. Female) & $1.862(0.971-3.572)$ & 0.061 & & \\
\hline $\begin{array}{l}\text { Baseline hemoglobin, } \\
\mathrm{g} / 1(<90 \text { vs. } \geq 90)\end{array}$ & $0.504(0.180-1.410)$ & 0.192 & & \\
\hline $\begin{array}{l}\text { Tumor location } \\
\text { (Right-sided vs. } \\
\text { Left-sided) }\end{array}$ & $2.034(1.114-3.716)$ & 0.021 & $\begin{array}{l}1.902(1.038-3 . \\
485)\end{array}$ & 0.038 \\
\hline $\begin{array}{l}\text { Tumor size, } \mathrm{cm}(>4 \text { vs. } \\
\leq 4)\end{array}$ & $0.843(0.460-1.547)$ & 0.582 & & \\
\hline $\begin{array}{l}\text { Differentiation (Poor vs. } \\
\text { Well/moderate) }\end{array}$ & $1.254(0.653-2.407)$ & 0.497 & & \\
\hline T stage (T4 vs. T1-3) & $1.926(1.022-3.628)$ & 0.043 & $\begin{array}{l}1.949(1.028-3 . \\
697)\end{array}$ & 0.041 \\
\hline $\begin{array}{l}\text { Number of resected } \\
\text { lymph nodes }(<12 \text { vs. } \geq \\
12)\end{array}$ & $1.097(0.563-2.138)$ & 0.786 & & \\
\hline N stage (N2 vs. N1) & $1.885(1.031-3.445)$ & 0.039 & $\begin{array}{l}1.773(0.962-3 . \\
270)\end{array}$ & 0.066 \\
\hline $\begin{array}{l}\text { Preoperative CEA, } \\
\mathrm{ng} / \mathrm{ml}(>5 \text { vs. } \leq 5)\end{array}$ & $0.980(0.534-1.796)$ & 0.947 & & \\
\hline $\begin{array}{l}\text { Preoperative CA19-9, } \\
\mathrm{U} / \mathrm{ml}(>24 \text { vs. } \leq 24)\end{array}$ & $2.231(1.224-4.067)$ & 0.009 & $\begin{array}{l}2.081(1.137-3 . \\
808)\end{array}$ & 0.017 \\
\hline $\begin{array}{l}\text { Adjuvant } \\
\text { chemotherapy, number } \\
\text { of cycles }(8 \text { vs. }<8)\end{array}$ & $0.510(0.279-0.930)$ & 0.028 & $\begin{array}{l}0.510(0.297-0 . \\
934)\end{array}$ & 0.029 \\
\hline
\end{tabular}

B

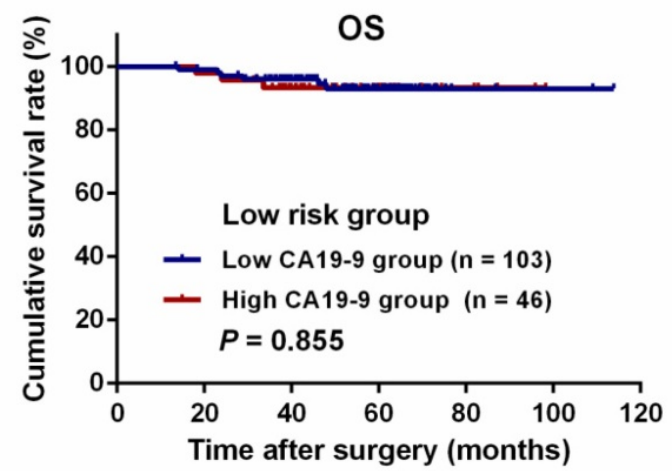

C

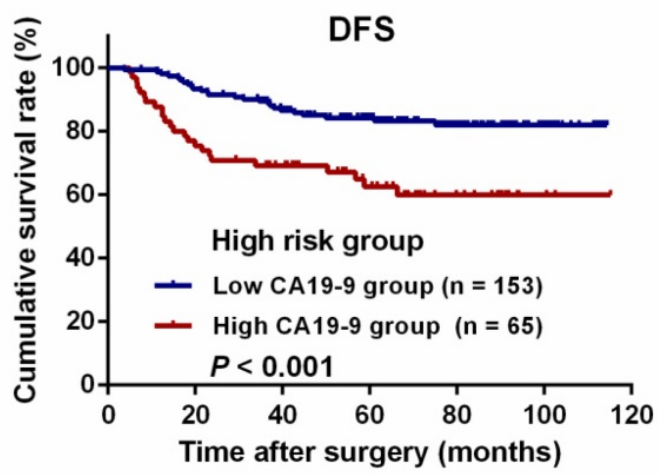

D

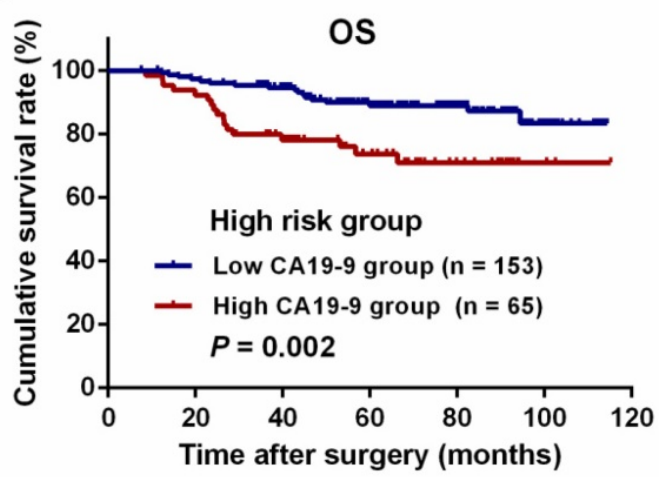

Figure 4. Kaplan-Meier curves for the comparison of long-term survival rates based on different preoperative serum carbohydrate antigen 19-9 (CA19-9) levels in patients stratified by high and low risk. (A) Disease-free survival (DFS) of patients with low-risk colon cancer. (B) Overall survival (OS) of patients with low-risk colon cancer. (C) DFS of patients with high-risk colon cancer. (D) OS of patients with high-risk colon cancer. 
Previous studies identified that CA19-9 was a prognostic factor for colorectal cancer in that patients with elevated baseline CA19-9 probably showed a high risk of postoperative metastasis, which contributed to a worse survival rate[19, 20]. Our results were consistent with those of these previous reports; we showed that patients with a high CA19-9 level presented a significantly increased incidence of postoperative metastasis $(32.4 \%$ vs. $15.2 \%, \mathrm{P}<0.001)$ compared with patients with a low CA19-9 level. CA19-9 clearly belongs to a cell surface glycoprotein and is involved in cellular adhesion. Cancer cells expressing this protein may present a greater metastatic and invasive potential [21, 22]. Moreover, the CA19-9 monosialoganglioside may be involved in the occurrence of tumor cell-induced platelet aggregation, which promotes the process of distant metastases in CRC [23]. Based on the involvement of CA19-9 in tumorigenesis, the malignant biological behavior of elevated CA19-9 might contribute to tumor metastasis and chemotherapeutic resistance.

With respect to the metastatic pattern, we found that high preoperative levels of CA19-9 indicated a higher incidence of lung metastasis $(12.6 \%$ vs. $3.9 \%, \mathrm{P}$ $=0.002)$ and abdominopelvic metastasis $(10.8 \%$ vs. $3.5 \%, \mathrm{P}=0.006$ ) than did low preoperative levels of CA19-9. As shown in our results, high CA19-9 levels were significantly associated with poorly differentiated and undifferentiated tumors. According to the results of a population-based study, poor differentiation, which is a feature of aggressive and advanced tumors, was identified as a high-risk factor for peritoneal carcinomatosis of colorectal origin [24, 25]. In line with our results, a previous study reported that high levels of CA19-9 were associated with a higher risk of lung metastasis and that the detection of preoperative CA19-9 levels might help predict lung metastasis [26, 27]. Furthermore, our results revealed that the high-CA19-9 group had a threefold higher cumulative incidence of lung metastasis than did the low-CA19-9 group $(12.0 \%$ vs. $3.9 \%, \mathrm{P}<0.001)$. Although the mechanisms underlying the increase in the incidence of lung metastasis remain unclear, early postoperative screening for lung metastasis with chest CT in patients with high levels of CA19-9 should be required. Early surveillance by a CT scan within 6 months after curative resection may be helpful for the treatment of stage III disease; salvage surgery for disease recurrence could thus be performed in a timely manner [28].

Interestingly, our result indicated that CA19-9 had a different impact on survival in high- and low-risk patients. There were no differences in the 3 -year survival rate among the low-risk patients with different CA19-9 levels, while among the high-risk patients, the survival rates were significantly worse in those with high CA19-9 levels than in those with low CA19-9 levels. This difference could be mainly attributed to the disparate characteristics between colon cancer patients with different risk levels. In general, patients with advanced disease were more likely to suffer from aggressive tumors, a high tumor burden, and aggravated immunosuppression [8, 29]. Additionally, previous studies have demonstrated that serum CA19-9 levels could accurately reflect tumor metabolism and tumor burden and thus may be suitable for monitoring treatment response and disease progression of pancreatic cancer [30, 31]. Therefore, the preoperative serum CA19-9 level was more sensitive for predicting survival outcomes in patients with high-risk colon cancer.

Our present study also reveals that the completion of oxaliplatin-containing adjuvant chemotherapy was a protective factor for 3-year OS in all patients. However, the possible efficacy of the treatment choice was associated with multiple factors, including patient age, comorbidities, compliance to therapy, and disease status [32-34]. Recently, the results of the International Duration Evaluation of Adjuvant Therapy (IDEA) project recommended that the division of stage III colon cancer into a low-risk (T1-3N1) and a high-risk (T4N1-2 or TanyN2) group was necessary; this division could improve adjuvant treatment selection for the patients [35]. Patients in the high-risk group should be given a full 6 months of the FOLFOX or XELOX regimen [16]. Nevertheless, TNM stage was not sufficient to warrant the division of the patients with different survival rates and chemotherapeutic benefits. Serum CA19-9 level was identified as a biological characteristic that could be incorporated into a standard TNM staging system to further refine risk stratification and predict benefits in OS from adjuvant chemotherapy in patients with resected pancreatic cancer [36]. Once patients were found as having high-risk disease with a high serum CA19-9 level, the intensified or full course of adjuvant chemotherapy should be strongly recommended. Besides, serum CA19-9 measurement is very easily performed in clinical settings and has a low cost. Therefore, we recommend that the preoperative serum CA19-9 level should be applied as a prognostic tool in clinical practice in order to provide useful information for therapeutic choices for patients with stage III colon cancer.

Some limitations of this study should be acknowledged. First, this retrospective study was conducted with an uncontrolled methodology with a limited number of patients from a single center. Second, the lack of the validation for the main 
findings and conclusions was another limitation of the study. Therefore, the findings need to be validated in multiple-center or larger cohort studies in the future. Third, the short duration of follow-up was insufficient to evaluate 5-year survival outcomes. This limitation may have led to an underestimation of the prognostic impact of serum CA19-9 level on long-term survival. In addition, our study assessed the CA19-9 level at a single time point before surgery, but the relative changes in the CA19-9 level during adjuvant chemotherapy and follow-up were not evaluated. Previous studies have reported that a decrease in the CA19-9 level at week 8 can serve as an early marker for predicting chemotherapeutic efficacy [37]. Moreover, molecular features of tumors, such as microsatellite instability (MSI), CpG island methylator phenotype (CIMP) level, and BRAF and KRAS mutational status, were not evaluated in this study; an analysis of the association of CA19-9 with these molecular markers would help us further understand the impact of CA19-9 on tumorigenesis in colon cancer. Despite these limitations, our findings suggested that the serum CA19-9 level should be considered a routine preoperative measurement for patients with stage III colon cancer.

\section{Conclusion}

The preoperative serum CA19-9 level is an independent prognostic factor for patients with stage III colon cancer undergoing curative resection followed by adjuvant chemotherapy. Measurement of the preoperative serum CA19-9 level has clinical implications for risk stratification and the individualization of the follow-up procedures as well as for adjuvant therapeutic strategies.

\section{Acknowledgments}

We deeply appreciate the help from all our colleagues at the Department of Colorectal Surgery, Sun Yat-sen University Cancer Center, who treated patients involved in the current study. We also thank Yixin Zhao, Jiali Chen, and Diefei Liao for offering their valuable insights. The authenticity of this article has been validated by uploading the key raw data onto the public Research Data Deposit platform (www.researchdata.org.cn) under the approval number RDDA2018000757.

\section{Funding}

This work was funded by grants from the National Natural Science Foundation of China (grant no. 81772595), Sun Yat-sen University Clinical Research 5010 Program (2015024) and Sun Yat-sen University Clinical Research 5010 Program (2013013).

\section{Availability of data and materials}

The datasets analysed during the current study were available from the corresponding author on reasonable request. Anyone who is interested in the information should contact fanwh@sysucc.org.cn and panzhzh@sysucc.org.cn.

\section{Competing Interests}

The authors have declared that no competing interest exists.

\section{References}

1. Siegel RL, Miller KD, Jemal A. Cancer statistics, 2018. Ca-A Cancer Journal For Clinicians. 2018;68(1):7-30.

2. Chen $W$, Zheng $R$, Baade $P$, Zhang $S$, Zeng $H$, Bray F, et al. Cancer statistics in China, 2015. Ca-A Cancer Journal For Clinicians. 2016;66(2):115-132.

3. Gunderson LL, Jessup JM, Sargent DJ, Greene FL, Stewart AK. Revised TN categorization for colon cancer based on national survival outcomes data. Journal of Clinical Oncology. 2010;28(2):264-171.

4. Chen VW, Hsieh MC, Charlton ME, Ruiz BA, Karlitz J, Altekruse SF, et al. Analysis of stage and clinical/prognostic factors for colon and rectal cancer from SEER registries: AJCC and collaborative stage data collection system. Cancer. 2014;120 Suppl 23:3793-3806.

5. Labianca R, Nordlinger B, Beretta GD, Mosconi S, Mandala M, Cervantes A, et al. Early colon cancer: ESMO Clinical Practice Guidelines for diagnosis, treatment and follow-up. Annals of Oncology. 2013;24 Suppl 6:vi64-72.

6. Shah MA, Renfro LA, Allegra CJ, Andre T, de Gramont A, Schmoll HJ, et al. Impact of Patient Factors on Recurrence Risk and Time Dependency of Oxaliplatin Benefit in Patients With Colon Cancer: Analysis From Modern-Era Adjuvant Studies in the Adjuvant Colon Cancer End Points (ACCENT) Database. Journal of Clinical Oncology. 2016;34(8):843-853.

7. Siegel R, Miller K, Fedewa S, Ahnen D, Meester R, Barzi A, et al. Colorectal cancer statistics, 2017. Ca-A Cancer Journal For Clinicians. 2017;67(3):177-193.

8. Peng J, Zhang R, Zhao Y, Wu X, Chen G, Wan D, et al. Prognostic value of preoperative prognostic nutritional index and its associations with systemic inflammatory response markers in patients with stage III colon cancer. Chinese Journal of Cancer. 2017;36(1):96

9. van Erning FN, Creemers GJ, De Hingh IH, Loosveld OJ, Goey SH, Lemmens VE. Reduced risk of distant recurrence after adjuvant chemotherapy in patients with stage III colon cancer aged 75 years or older. Annals of oncology. 2013;24(11):2839-44.

10. Koprowski H, Herlyn M, Steplewski Z, Sears H. Specific antigen in serum of patients with colon carcinoma. Science. 1981;212(4490):53-55.

11. Marrelli D, Caruso S, Pedrazzani C, Neri A, Fernandes E, Marini M, et al. CA19-9 serum levels in obstructive jaundice: clinical value in benign and malignant conditions. American Journal of Surgery. 2009;198(3):333-339.

12. De Marchi G, Paiella S, Luchini C, Capelli P, Bassi C, Frulloni L. Very high serum levels of CA 19-9 in autoimmune pancreatitis: Report of four cases and brief review of literature. Journal Of Digestive Diseases . 2016;17(10):697-702.

13. Chan A, Prassas I, Dimitromanolakis A, Brand R, Serra S, Diamandis E, et al. Validation of biomarkers that complement CA19.9 in detecting early pancreatic cancer. Clinical Cancer Research. 2014;20(22):5787-5795.

14. Chen J, Zhao T, Jia S, Zhou S, Zhou L, Wang S, et al. High Expression of P38a and Preoperative Carbohydrate Antigen 19-9 Indicate Poor Prognosis in Patients with Pancreatic Ductal Adenocarcinoma. Journal of Cancer. 2018;9(4):650-659.

15. Grunnet M, Christensen I, Lassen U, Jensen L, Lydolph M, Knox J, et al. Decline in CA19-9 during chemotherapy predicts survival in four independent cohorts of patients with inoperable bile duct cancer. European Journal of Cancer. 2015;51(11):1381-1388.

16. Benson A, Venook A, Al-Hawary M, Cederquist L, Chen Y, Ciombor K, et al. NCCN Guidelines Insights: Colon Cancer, Version 2.2018. Journal of the National Comprehensive Cancer Network . 2018;16(4):359-369.

17. Stiksma J, Grootendorst D, van der Linden P. CA 19-9 as a marker in addition to CEA to monitor colorectal cancer. Clinical Colorectal Cancer. 2014;13(4):239-244.

18. Abe S, Kawai K, Ishihara S, Nozawa H, Hata K, Kiyomatsu T, et al. Prognostic impact of carcinoembryonic antigen and carbohydrate antigen 19-9 in stage IV colorectal cancer patients after R0 resection. Journal of Surgical Research. 2016;205(2):384-392.

19. Lu Z, Peng J, Wang Z Pan $Z$ Yuan $Y$, Wan $D$, et al. High preoperative serum CA19-9 level is predictive of poor prognosis for patients with colorectal liver oligometastases undergoing hepatic resection. Medical Oncology. 2016;33(11):121.

20. Sato H, Kotake $\mathrm{K}$, Sugihara $\mathrm{K}$, Takahashi H, Maeda K, Uyama I, et al. Clinicopathological Factors Associated with Recurrence and Prognosis after R0 Resection for Stage IV Colorectal Cancer with Peritoneal Metastasis. Digestive Surgery. 2016;33(5):382-391. 
21. Scarà S, Bottoni P, Scatena R. CA 19-9: Biochemical and Clinical Aspects. Advances in Experimental Medicine and Biology. 2015;867:247-260.

22. Swords D, Firpo M, Scaife C, Mulvihill S. Biomarkers in pancreatic adenocarcinoma: current perspectives. Oncotargets and Therapy. 2016;9:7459-7467.

23. Martini F, Guadagni F, Lenti L, D'Alessandro R, Aloe S, Roselli M, et al. CA 19-9 monosialoganglioside content of human colorectal tumor cells correlates with tumor cell-induced platelet aggregation. Anticancer Research. 2000;20(3A):1609-1614.

24. Soliman BG, Karagkounis G, Church JM, Plesec T, Kalady MF. Mucinous Histology Signifies Poor Oncologic Outcome in Young Patients With Colorectal Cancer. Diseases of the Colon and Rectum. 2018;61(5):547-553.

25. Lemmens VE, Klaver YL, Verwaal VJ, Rutten HJ, Coebergh JW, de Hingh IH. Predictors and survival of synchronous peritoneal carcinomatosis of colorectal origin: a population-based study. International Journal of Cancer. 2011;128(11):2717-2725.

26. Lin $\mathrm{P}$, Lin J, Lin C, Wang H, Yang S, Jiang J, et al. Carbohydrate antigen 19-9 is a valuable prognostic factor in colorectal cancer patients with normal levels of carcinoembryonic antigen and may help predict lung metastasis. International Journal of Colorectal Disease. 2012;27(10):1333-1338.

27. Yamaguchi E, Makino Y, Sato T, Uchida M, Harada Y, Maruyama R. CA19-9-producing lung metastasis after surgery for papillary thyroid carcinoma: report of a case. Surgery Today. 2014;44(11):2157-2161.

28. Liu G, Tang J, Wen S, Cao H, An X, Cai P, et al. Is early surveillance with CT scan necessary in patients with stage II/III colorectal cancer: a retrospective study. J Surg Oncol. 2013;108(8):568-71.

29. Roshani R, McCarthy F, Hagemann T. Inflammatory cytokines in human pancreatic cancer. Cancer Letters. 2014;345(2):157-163.

30. Xu H, Chen T, Wang W, Wu C, Liu C, Long J, et al. Metabolic tumour burden assessed by ${ }^{18} \mathrm{~F}-\mathrm{FDG}$ PET/CT associated with serum CA19-9 predicts pancreatic cancer outcome after resection. European Journal of Nuclear Medicine and Molecular Imaging. 2014;41(6):1093-1102.

31. Shi S, Ji S, Qin Y, Xu J, Zhang B, Xu W, et al. Metabolic tumor burden is associated with major oncogenomic alterations and serum tumor markers in patients with resected pancreatic cancer. Cancer Lett. 2015;360(2):227-233.

32. McCleary N, Meyerhardt J, Green E, Yothers G, de Gramont A, Van Cutsem E, et al. Impact of age on the efficacy of newer adjuvant therapies in patients with stage II/III colon cancer: findings from the ACCENT database. Journal of Clinical Oncology. 2013;31(20):2600-2606.

33. Baretti M, Rimassa L, Personeni N, Giordano L, Tronconi M, Pressiani T, et al. Effect of Comorbidities in Stage II/III Colorectal Cancer Patients Treated With Surgery and Neoadjuvant/Adjuvant Chemotherapy: A Single-Center, Observational Study. Clinical Colorectal Cancer. 2018. pii: S1533-0028(17)30382-1.

34. Lin C, Bruinooge S, Kirkwood M, Olsen C, Jemal A, Bajorin D, et al. Association Between Geographic Access to Cancer Care, Insurance, and Receipt of Chemotherapy: Geographic Distribution of Oncologists and Travel Distance. Journal Clinical Oncol. 2015;33(28):3177-3185.

35. Grothey A, Sobrero A, Shields A, Yoshino T, Paul J, Taieb J, et al. Duration of Adjuvant Chemotherapy for Stage III Colon Cancer. The New England Journal of Medicine. 2018;378(13):1177-1188

36. Liu L, Xu H, He M, Wang W, Wang W, Wu C, et al. A novel scoring system predicts postsurgical survival and adjuvant chemotherapeutic benefits in patients with pancreatic adenocarcinoma: Implications for AJCC-TNM staging. Surgery. 2018:163(6):1280-1294.

37. Chiorean $E$, Von Hoff $D$, Reni M, Arena F, Infante J, Bathini V, et al. CA19-9 decrease at 8 weeks as a predictor of overall survival in a randomized phase III trial (MPACT) of weekly nab-paclitaxel plus gemcitabine versus gemcitabine alone in patients with metastatic pancreatic cancer. Annals of Oncol. 2016;27(4):654-660 